\title{
Nanoscale Size Effects on Crystallization Kinetics of Metallic Glass Nanorods by In Situ TEM
}

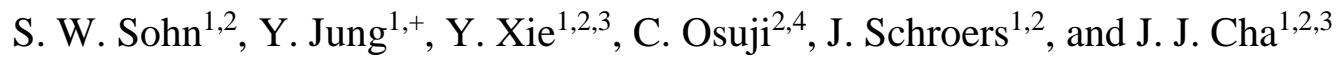

1. Department of Mechanical Engineering and Materials Science, Yale University, New Haven, USA

2. Center for Research on Interface Structures and Phenomena, Yale University, New Haven, USA

3. Energy Sciences Institute, Yale West Campus, West Haven, USA

4. Department of Chemical \& Environmental Engineering, Yale University, New Haven, USA

+. Present address: Nanoscience Technology Center, University of Central Florida, Orlando, USA

Metallic glasses are attractive for a variety of applications due to their attractive materials properties. Their lack of long-range order gives rise to superior mechanical and, in some cases, more desirable electrical and chemical properties compared to crystalline counterparts [1]. Making metallic glasses into nanostructures provides additional functionality not possible in bulk forms through nanoscale surface patterning. However, their crystallization behavior at the nanoscale is not well understood, limiting fabrication of small length scale features achievable with the current thermoplastic molding fabrication technique [2]. Scientifically, metallic glasses are a model system to study long-standing questions in nucleation and crystallization of solids owing to their simple metallic bonds, easily accessible slow crystallization kinetics, accurate control of their chemical compositions [3], and nanostructure fabrication that has recently been developed $[2,4]$.

By in situ heating size-controlled Pt-based metallic glass nanorods inside a transmission electron microscope (Figure 1), we directly show that the crystallization kinetics in nanoscale dimension deviates significantly from the bulk [5]. In particular, the crystallization kinetics slows down rapidly below $\sim 30$ nm (Figure 2) due to competing size-dependent factors: heterogeneous nucleation, enhanced apparent viscosity, and lower probability of nucleation in small samples. In particular, the enhanced viscosity is supported by direct observation of slowed grain growth (Figure 3). These results indicate a departure from the conventional continuum description of glassy materials and point to a nanoscale confinement effect when the sample size approaches the size scale of intrinsic flow units. Thus, our findings address a basic yet important question regarding size-dependent effects in the crystallization kinetics of metallic glasses.

Technologically, our findings provide insight into the crystallization of supercooled metallic liquids at relevant length scales. This is crucial as the thermoplastic formability of metallic glasses spans over ten orders of magnitude in length scales from meters to angstroms, allowing multi-scale, hierarchical structuring of metallic glasses for novel surface properties. Our findings offer critical new knowledge on how to avoid crystallization during nanomolding of metallic glasses, thus retaining the attractive properties of these multicomponent alloys in their amorphous state. [6]

\section{References:}

[1] A. L. Greer, Science 267 (1995), p.1947-1953.

[2] G. Kumar, H. X. Tang, J. Schroers, Nature 457 (2009), p.868-872. 
[3] S. Ding, et al, Nature Materials 13 (2014) p. 494 - 500.

[4] Y. Liu et al, Nature Communications 6 (2015) 7043.

[5] S. Sohn et al, Nature Communications 6 (2015) 8157.

[6] The authors acknowledge funding from the National Science Foundation MRSEC DMR 1119826.
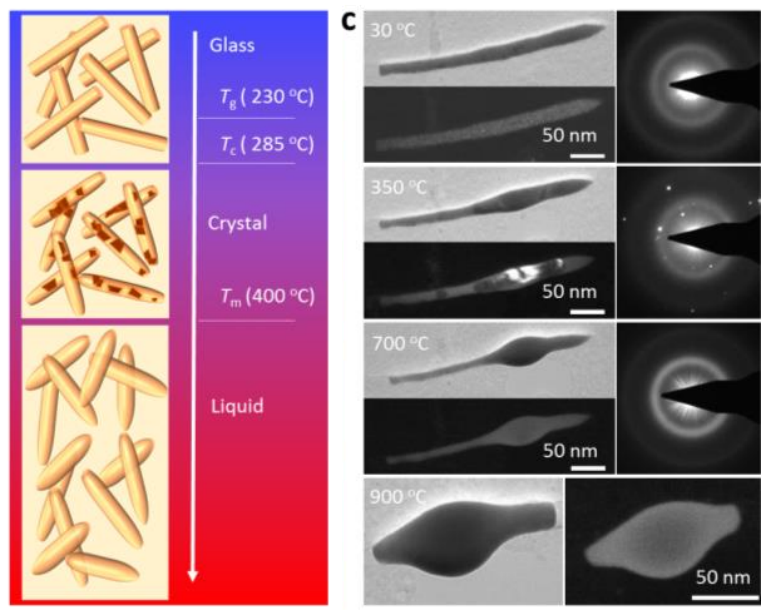

Figure 1. In situ crystallization of a metallic glass nanorod inside a TEM. Left: Schematic with bulk values for glass transition $\left(T_{g}\right)$, crystallization $\left(T_{c}\right)$, and melting $\left(\mathrm{T}_{\mathrm{m}}\right)$ temperatures. Right: observed crystallization of a metallic glass nanorod in bright-field and dark-field TEM images and diffraction patterns.
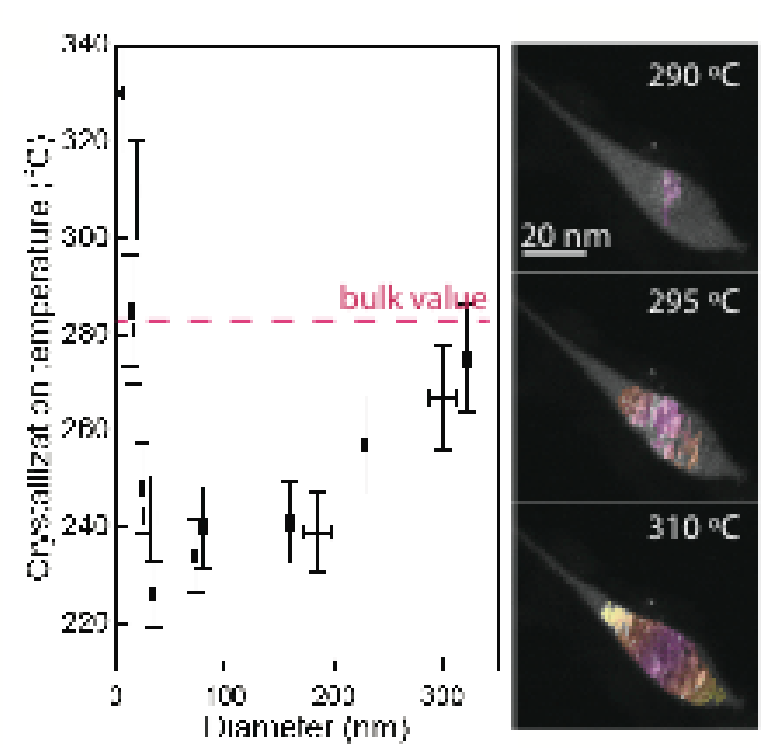

Figure 2. Nanoscale effects in crystallization kinetics. Left: Measured crystallization temperatures as a function of nanorod diameter. Right: A tapered nanorod initially crystallizes from the widest region of the rod.

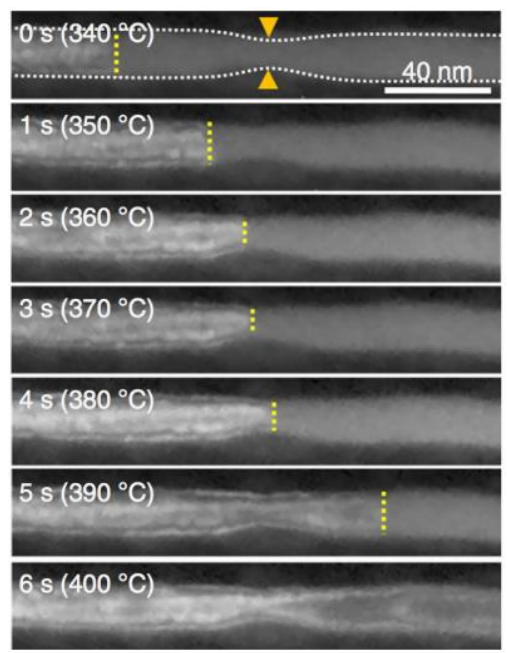

Figure 3. Observation of slowed grain growth through the narrower region of the nanorod. The yellow dotted line indicates the grain boundary. 